\title{
Prevalence of Suboptimal Health Status and the Relationships between Suboptimal Health Status and Lifestyle Factors among Chinese Adults Using a Multi-Level Generalized Estimating Equation Model
}

\author{
Tao $\mathrm{Xu}{ }^{1, *} \mathbb{0}$, Guangjin $\mathrm{Zhu}^{2}$ and Shaomei Han ${ }^{1}$ \\ 1 Department of Epidemiology and Statistics, Institute of Basic Medical Sciences, Chinese Academy of Medical \\ Sciences and School of Basic Medicine, Peking Union Medical College, Beijing 100005, China; \\ hansm1@vip.sina.com \\ 2 Department of Physiopathology, Institute of Basic Medical Sciences, Chinese Academy of Medical Sciences \\ and School of Basic Medicine, Peking Union Medical College, Beijing 100005, China; zgj_1942@tom.com \\ * Correspondence: xutaosd@126.com
}

Received: 2 January 2020; Accepted: 22 January 2020; Published: 25 January 2020

\begin{abstract}
This study examined the prevalence of suboptimal health among Chinese adults based on a large-scale national survey and clarified the relationship between suboptimal health and lifestyle factors. We used multi-level generalized estimating equation models to examine the relationships between suboptimal health and lifestyle factors. Of the 48,978 respondents, 34,021 reported one or more suboptimal health symptoms, giving a suboptimal health status prevalence of $69.46 \%$. After controlling for the cluster effect of living areas and confounding effect of demographic characteristics, factors associated with suboptimal health were: current smoking (odds ratio $(\mathrm{OR})=1.083,95 \%$ confidence interval (CI): 1.055-1.111), drinking alcohol (OR = 1.075, 95\% CI: 1.025-1.127), family history of disease ( $\mathrm{OR}=1.203,95 \% \mathrm{CI}: 1.055-1.111)$, sleeping $<6 \mathrm{~h}$ per day $(\mathrm{OR}=1.235,95 \% \mathrm{CI}$ : $1.152-1.256)$, poor sleep quality (OR $=1.594,95 \% \mathrm{CI}: 1.515-1.676)$, stress $(\mathrm{OR}=1.588,95 \% \mathrm{CI}$ : 1.496-1.686), negative life events ( $\mathrm{OR}=1.114,95 \% \mathrm{CI}: 1.045-1.187)$, unhealthy diet choices (OR $=1.093$, 95\% CI: 1.033-1.156), and not regularly having meals at fixed hours (OR $=1.231,95 \%$ CI: $1.105-1.372$ ). Respondents who exercised regularly had lower odds of having suboptimal health status ( $\mathrm{OR}=0.913$, 95\% CI: 0.849-0.983). Suboptimal health has become a serious public health challenge in China. The health status of the population could be effectively improved by improving lifestyle behaviors.
\end{abstract}

Keywords: suboptimal health; prevalence; lifestyle; generalized estimating equation model

\section{Introduction}

The World Health Organization defines health in its broader sense as a state of complete physical, mental, and social well-being, rather than merely as the absence of disease or infirmity [1]. With advancing understanding of health, this definition has expanded to consider suboptimal health status. Although there is currently no common definition for suboptimal health status, people usually consider suboptimal health as a borderline state between health and disease, characterized by declines in vitality, physiological function, and the capacity for adaptation, and including medically undiagnosed or functional somatic syndromes [2,3]. In China, rapid economic development has meant that many people face pressures from their work and home lives that may develop into suboptimal health status. Globally, suboptimal health has attracted increasing attention from medical professionals as an important public health issue [4,5]. It is important to properly assess suboptimal health and explore the factors associated with suboptimal health to prevent diseases and improve human health. 
Previous epidemiological surveys showed that $55 \%-75 \%$ of China's population experienced suboptimal health [6-8].

Lifestyle behaviors are important factors affecting diseases and health. Many lifestyle behaviors influence a person's ability to maintain their energy balance over the long term. For example, duration of sleep may influence energy consumption, energy expenditure, or both $[9,10]$. Unhealthy lifestyles are also closely related to many chronic diseases [11-14]. Chen reported that in the general population, major risk factors for suboptimal health status included poor stress management, poor self-actualization, lack of exercise, and poor interpersonal relationships [15].

Despite the increasing availability of information on this topic, more evidence is needed regarding the prevalence of suboptimal health among representative Chinese populations that cover a broad age range and include different minorities and regions. To date, most estimates of suboptimal health status have been based on a single province or small sample size, and could not represent the general Chinese adult population $[6-8,16]$. Little is known about the current prevalence of suboptimal health status in China based on large-scale representative national samples. Furthermore, people living in the same area may be likely to suffer from the effects of similar living circumstances, which may influence the accuracy and generalizability of data on the relationship between suboptimal health and lifestyle. However, current studies focused on suboptimal health have not considered and adjusted for the cluster effect of living area, which has a confusing influence on the results of studies investigating suboptimal health. Specifically, this study aimed to examine the prevalence of suboptimal health among Chinese adults. We covered a broad age range using data from a large-scale cross-sectional national survey, and clarified the relationships between suboptimal health status and lifestyle factors using multi-level generalized estimating equation (GEE) models controlled for the cluster effect of living area.

\section{Material and Methods}

\subsection{Sample and Participants}

This survey was conducted in six provinces or autonomous regions of China from 2007 to 2011: Heilongjiang province, Hunan province, Yunnan province, Inner Mongolia autonomous region, Sichuan province, and Ningxia Hui autonomous region. China is a vast multi-ethnic country with huge regional differences, populated by Han and 55 ethnic minorities. These six provinces or autonomous regions were selected using convenience sampling to cover a variety of municipal regions and include sufficient minority respondents. In each selected province or autonomous region, a two-stage cluster sampling method was used to recruit eligible respondents. Two or three cities were selected based on their population and economic conditions using a simple random sampling method. Next, dozens of communities were selected within each city using a cluster random sampling method based on population density. All residents of the selected communities were considered eligible for the survey if they were aged $\geq 18$ years, were not suffering from serious chronic diseases, and had not run a high fever in the past 15 days. After signing informed consent forms, all respondents voluntarily attended temporary physical examination centers to take part in the survey. The survey was approved by the Review Board of the Institute of Basic Medical Sciences, Chinese Academy of Medical Sciences (No. 005-2008).

\subsection{Suboptimal Health Assessment}

The self-rating suboptimal health scale developed using a Delphi method by researchers from Medical College of Jinan University was used to assess the suboptimal health status of the community populations [17]. Previous reports showed that this scale had good reliability and repeatability [17-19]. The scale includes 18 symptom items grouped in six dimensions: physical symptoms, psychological symptoms, vigor, social adaptability, immunity, and going to hospital. The physical symptoms dimension contains five suboptimal health symptom items: fatigue, headache or dizziness, tinnitus, 
numbness or stiffness in the shoulders or legs, and a sense of pharyngeal foreign bodies. The psychological symptoms dimension contains six suboptimal health symptom items: upset, loneliness, inattention, anxiety, dreaminess, and forgetfulness. The vigor dimension contains three suboptimal health symptom items: decreased vitality, disinterest in surroundings and moodiness. The social adaptability dimension contains two suboptimal health symptom items: feeling tired at work and incompatibility with coworkers. The immunity dimension contains one suboptimal health symptom item: susceptibility to flu or other diseases. The going to hospital dimension contains one suboptimal health symptom item: the feeling of suffering from undiagnosed diseases. Respondents that had experienced one or more symptom item for more than one month in the past year were considered as having suboptimal health status.

\subsection{Definition of Covariates}

Covariates included demographic characteristics (e.g., age, gender, occupation, marital status, educational level) and lifestyle factors such as smoking, alcohol drinking, ethnicity, family history of cardiovascular or cerebrovascular diseases, body mass index (BMI), sleep duration, sleep quality, stress, life events, hypertension, exercise, diet choices, and meal times.

Respondents were categorized into three age groups: youth (18-34 years), middle aged (35-59 years), and older adults ( $\geq 60$ years old). Occupation was classified as blue-collar worker and "white-collar worker" (e.g., teacher, doctor, other professionals, students, governmental and institute employees). Marital status was categorized as married, single, divorced, or widowed. Educational level was categorized into three groups: primary school, middle school, and college. Ethnicity included Han, Yi, Miao, Mongolia, Tibetan, Korean, Hui, Tujia, and others. BMI was defined as weight in $\mathrm{kg}$ divided by height in $\mathrm{m}^{2}$. Weight was measured to the nearest $0.1 \mathrm{~kg}$ and height was measured to the nearest $1 \mathrm{~mm}$. According to Chinese guidelines for the prevention and control of adult overweight and obesity and the Working Group on Obesity in China criteria [20], normal weight was defined as a BMI $<24 \mathrm{~kg} / \mathrm{m}^{2}$, overweight as a BMI of $24-27.9 \mathrm{~kg} / \mathrm{m}^{2}$, and obesity as a BMI $\geq 28 \mathrm{~kg} / \mathrm{m}^{2}$.

Sleep duration was dichotomized into $>6 \mathrm{~h}$ of sleep and $<6 \mathrm{~h}$ of sleep. Respondents were also asked to self-report whether they had good sleep quality. Stress was defined as self-perceived economic, life, or work stress. Life events were defined by whether the respondent had recently experienced negative or positive events. Negative events included loss of job, retirement, loss of crops/business failure, burglary, marital separation/divorce, other major intra-family conflict, major personal injury or illness, violence, death of a spouse, death/major illness of another close family member, and other major stressors. Positive events included events such as the wedding of a family member, new job, or a birth in the family. Diet choice was defined as whether the respondent had unhealthy diet choice preferences, such as food that was highly spicy, sweet, salty, or greasy. Meal time was used to indicate whether the respondent regularly had breakfast, lunch, and dinner at fixed times.

Blood pressure was measured in the morning after respondents had rested for $5 \mathrm{~min}$ in a seated position with their back supported, feet on the floor, and right arm supported with the cubit fossa at heart level. The appropriate cuff was chosen based on the respondent's arm circumference. OMRON HEM-7000 electronic sphygmomanometers (OMRON Health-Care, Kyoto, Japan) were used to measure blood pressure. Hypertension was defined as not being on antihypertensive medications and having systolic blood pressure of $\geq 140 \mathrm{mmHg}$ or diastolic blood pressure of $\geq 90 \mathrm{mmHg}$ [21,22].

\subsection{Quality Control}

This study adhered to strict quality control standards. All researchers were trained based on a training manual. A preliminary survey was conducted to confirm that the investigators were conducting the survey correctly. Trained medical professionals conducted the survey and respondent interviews. All case report forms were double-checked to guarantee the authenticity and accuracy of raw data. The survey database was constructed with EPI3.02 software (EpiData Association, Odense, 
Denmark), with data input checked twice by two data managers to guarantee the accuracy and integration of the data.

\subsection{Statistical Analysis}

Statistical analysis was performed with SAS9.4 software (SAS institute Inc., Cary, NC, USA). A two-tailed $p$-value $<0.05$ was defined as statistically significant. Continuous data were described using mean and standard deviation when normally distributed, and median (lower quartile, upper quartile) when the distribution was skewed. Categorical data were described with number and percentage and compared using chi-square tests. Considering that respondents living in the same city were likely to suffer the effects of similar living circumstances, multi-level GEE models were used to examine the relationships between suboptimal health status and lifestyle factors to control for the cluster effect of living area and the confounding effect of demographic characteristics. Odds ratios and 95\% confidence intervals were used to assess the strength of these relationships.

\section{Results}

In total, 52,265 respondents signed an informed consent form and were willing to participate in this survey. Of these, 48,978 respondents completed all survey scales, giving a completion rate of $93.7 \%$.

The average age of all respondents was $44.3 \pm 16.8$ years, and $59.5 \%$ respondents were female. In total, $46.7 \%$ of respondents were white-collar workers, such as teachers, doctors, other professionals, students, and governmental and institute employees. Widowed or divorced respondents accounted for $5.45 \%$ of the sample. The majority of respondents $(74.9 \%)$ were of Han nationality. The percentages of regular smokers and drinkers were $22.5 \%$ and $22.3 \%$, respectively. The percentages of overweight and obese respondents were $31.2 \%$ and $12.0 \%$, respectively. In addition, 782 respondents reported feeling high levels of work, home, or economic stress, and 2058 respondents had recently experienced positive or negative life events. Just under half (45.87\%) of the respondents exercised regularly, $13.30 \%$ slept for $<6$ h per day, and only $28.33 \%$ reported good sleep quality. The majority of respondents $(67.67 \%)$ had special dietary preferences and $9.84 \%$ did not regularly have meals at fixed hours.

Of the 48,978 respondents, 34,021 reported one or more suboptimal health symptoms, giving a suboptimal health status prevalence rate of $69.46 \%$. The median number of suboptimal health symptoms was $2(0,5)$. The most common suboptimal health symptoms were forgetfulness $(38.53 \%)$, dreaminess (30.89\%), and headache or dizziness (30.10\%). Table 1 shows the prevalence of each the 18 suboptimal health symptoms for the whole sample, and by sex and age groups.

The average age of respondents with a suboptimal health status was $45.0 \pm 16.7$ years and that of respondents without suboptimal health was $42.6 \pm 17.0$ years. The prevalence rate of suboptimal health status was $65.02 \%$ for youths, $71.08 \%$ for middle-aged adults, and $72.48 \%$ for older adults. The prevalence rate of suboptimal health status for males $(67.74 \%)$ was lower than that for females $(72.67 \%)$ $(p<0.0001)$. The prevalence rate of suboptimal health status was statistically significantly different among various ethnicities; respondents from Tujia had the lowest prevalence of suboptimal health status $(60.21 \%)$ and those from Yi had the highest prevalence $(87.15 \%)$. Blue-collar workers had a higher prevalence $(73.71 \%)$ of suboptimal health status than their white-collar worker counterparts. The prevalence rate of suboptimal health status declined as educational level increased $(p<0.0001)$. The prevalence rate of suboptimal health status for widowed or divorced respondents (77.11\%) was significantly higher than that among married or single respondents. 
Table 1. Prevalence (\%) of 18 suboptimal health symptoms by sex and age groups.

\begin{tabular}{|c|c|c|c|c|c|c|}
\hline \multirow{2}{*}{ Items } & \multirow{2}{*}{$\begin{array}{c}\text { Total }(n= \\
48,978)\end{array}$} & \multicolumn{2}{|c|}{ Sex } & \multicolumn{3}{|c|}{ Age Groups } \\
\hline & & $\begin{array}{c}\text { Men }(n= \\
19,834)\end{array}$ & $\begin{array}{c}\text { Women }(n= \\
29,144)\end{array}$ & $\begin{array}{c}18-34 \text { y }(n= \\
15,430)\end{array}$ & $\begin{array}{c}35-59 \text { y }(n= \\
23,372)\end{array}$ & $\begin{array}{c}\geq 60 \text { y }(n= \\
10,176)\end{array}$ \\
\hline Fatigue & $\begin{array}{c}14,300 \\
(29.20 \%)\end{array}$ & $\begin{array}{c}5142 \\
(25.93 \%)\end{array}$ & $\begin{array}{c}9158 \\
(31.42 \%)\end{array}$ & $\begin{array}{c}4261 \\
(27.62 \%)\end{array}$ & $\begin{array}{c}7264 \\
(31.08 \%)\end{array}$ & $\begin{array}{c}2775 \\
(27.27 \%)\end{array}$ \\
\hline $\begin{array}{l}\text { Headache or } \\
\text { dizziness }\end{array}$ & $\begin{array}{c}14,740 \\
(30.10 \%)\end{array}$ & $\begin{array}{c}4474 \\
(22.56 \%)\end{array}$ & $\begin{array}{c}10,266 \\
(35.23 \%)\end{array}$ & $\begin{array}{c}3135 \\
(20.32 \%)\end{array}$ & $\begin{array}{c}8068 \\
(34.52 \%)\end{array}$ & $\begin{array}{c}3537 \\
(34.76 \%)\end{array}$ \\
\hline Tinnitus & $\begin{array}{c}8415 \\
(17.18 \%)\end{array}$ & $\begin{array}{c}3169 \\
(15.98 \%)\end{array}$ & $\begin{array}{c}5246 \\
(18.00 \%)\end{array}$ & $1248(8.09 \%)$ & $\begin{array}{c}4621 \\
(19.77 \%)\end{array}$ & $\begin{array}{c}2546 \\
(25.02 \%)\end{array}$ \\
\hline $\begin{array}{c}\text { Numbness } \\
\text { or stiffness } \\
\text { in the } \\
\text { shoulders or } \\
\text { legs }\end{array}$ & $\begin{array}{c}10,324 \\
(21.08 \%)\end{array}$ & $\begin{array}{c}3440 \\
(17.34 \%)\end{array}$ & $\begin{array}{c}6884 \\
(23.62 \%)\end{array}$ & $1498(9.71 \%)$ & $\begin{array}{c}5861 \\
(25.08 \%)\end{array}$ & $\begin{array}{c}2965 \\
(29.14 \%)\end{array}$ \\
\hline $\begin{array}{l}\text { A sense of } \\
\text { pharyngeal } \\
\text { foreign } \\
\text { bodies }\end{array}$ & $\begin{array}{c}8205 \\
(16.75 \%)\end{array}$ & $\begin{array}{c}3324 \\
(16.76 \%)\end{array}$ & $\begin{array}{c}4881 \\
(16.75 \%)\end{array}$ & $\begin{array}{c}2738 \\
(17.74 \%)\end{array}$ & $\begin{array}{c}4037 \\
(17.27 \%)\end{array}$ & $\begin{array}{c}1430 \\
(14.05 \%)\end{array}$ \\
\hline Upset & $\begin{array}{c}10,192 \\
(20.81 \%)\end{array}$ & $\begin{array}{c}3050 \\
(15.38 \%)\end{array}$ & $\begin{array}{c}7142 \\
(24.51 \%)\end{array}$ & $\begin{array}{c}3485 \\
(22.59 \%)\end{array}$ & $\begin{array}{c}4987 \\
(21.34 \%)\end{array}$ & $\begin{array}{c}1720 \\
(16.90 \%)\end{array}$ \\
\hline Loneliness & $4238(8.65 \%)$ & 1547 (7.80\%) & $2691(9.23 \%)$ & $\begin{array}{c}1890 \\
(12.25 \%)\end{array}$ & $1573(6.73 \%)$ & $775(7.62 \%)$ \\
\hline Inattention & $\begin{array}{c}9944 \\
(20.30 \%)\end{array}$ & $\begin{array}{c}3515 \\
(17.72 \%)\end{array}$ & $\begin{array}{c}6429 \\
(22.06 \%)\end{array}$ & $\begin{array}{c}3897 \\
(25.26 \%)\end{array}$ & $\begin{array}{c}4206 \\
(18.00 \%)\end{array}$ & $\begin{array}{c}1841 \\
(18.09 \%)\end{array}$ \\
\hline Anxiety & $\begin{array}{c}7064 \\
(14.42 \%)\end{array}$ & $\begin{array}{c}2323 \\
(11.71 \%)\end{array}$ & $\begin{array}{c}4741 \\
(16.27 \%)\end{array}$ & $\begin{array}{c}2513 \\
(16.29 \%)\end{array}$ & $\begin{array}{c}3272 \\
(14.00 \%)\end{array}$ & $\begin{array}{c}1279 \\
(12.57 \%)\end{array}$ \\
\hline Dreaminess & $\begin{array}{c}15,131 \\
(30.89 \%)\end{array}$ & $\begin{array}{c}4733 \\
(23.86 \%)\end{array}$ & $\begin{array}{c}10,398 \\
(35.68 \%)\end{array}$ & $\begin{array}{c}4478 \\
(29.02 \%)\end{array}$ & $\begin{array}{c}7544 \\
(32.28 \%)\end{array}$ & $\begin{array}{c}3109 \\
(30.55 \%)\end{array}$ \\
\hline Forgetfulness & $\begin{array}{c}18,872 \\
(38.53 \%)\end{array}$ & $\begin{array}{c}6601 \\
(33.28 \%)\end{array}$ & $\begin{array}{c}12,271 \\
(42.10 \%)\end{array}$ & $\begin{array}{c}4579 \\
(29.68 \%)\end{array}$ & $\begin{array}{c}9641 \\
(41.25 \%)\end{array}$ & $\begin{array}{c}4652 \\
(45.72 \%)\end{array}$ \\
\hline $\begin{array}{l}\text { Decreased } \\
\text { vitality }\end{array}$ & $\begin{array}{c}9872 \\
(20.16 \%)\end{array}$ & $\begin{array}{c}3645 \\
(18.38 \%)\end{array}$ & $\begin{array}{c}6227 \\
(21.37 \%)\end{array}$ & $\begin{array}{c}2366 \\
(15.33 \%)\end{array}$ & $\begin{array}{c}4643 \\
(19.87 \%)\end{array}$ & $\begin{array}{c}2863 \\
(28.13 \%)\end{array}$ \\
\hline $\begin{array}{l}\text { Disinterest in } \\
\text { surroundings }\end{array}$ & $\begin{array}{c}6514 \\
(13.30 \%)\end{array}$ & $\begin{array}{c}2361 \\
(11.90 \%)\end{array}$ & $\begin{array}{c}4153 \\
(14.25 \%)\end{array}$ & $\begin{array}{c}2248 \\
(14.57 \%)\end{array}$ & $\begin{array}{c}2928 \\
(12.53 \%)\end{array}$ & $\begin{array}{c}1338 \\
(13.15 \%)\end{array}$ \\
\hline Moodiness & $\begin{array}{c}7179 \\
(14.66 \%)\end{array}$ & $\begin{array}{c}2388 \\
(12.04 \%)\end{array}$ & $\begin{array}{c}47,911 \\
(16.44 \%)\end{array}$ & $\begin{array}{c}2761 \\
(17.89 \%)\end{array}$ & $\begin{array}{c}3215 \\
(13.76 \%)\end{array}$ & $\begin{array}{c}1203 \\
(11.82 \%)\end{array}$ \\
\hline $\begin{array}{l}\text { Feeling tired } \\
\text { at work }\end{array}$ & $\begin{array}{c}5279 \\
(10.78 \%)\end{array}$ & $\begin{array}{c}2048 \\
(10.33 \%)\end{array}$ & $\begin{array}{c}3231 \\
(11.09 \%)\end{array}$ & $\begin{array}{c}1753 \\
(11.36 \%)\end{array}$ & $\begin{array}{c}2627 \\
(11.24 \%)\end{array}$ & 899 (8.83\%) \\
\hline $\begin{array}{l}\text { Incompatibility } \\
\text { with } \\
\text { coworkers }\end{array}$ & $1588(3.24 \%)$ & $630(3.18 \%)$ & $958(3.29 \%)$ & $523(3.39 \%)$ & 765 (3.27\%) & $300(2.95 \%)$ \\
\hline $\begin{array}{l}\text { Susceptibility } \\
\text { to flu or } \\
\text { other } \\
\text { diseases }\end{array}$ & $\begin{array}{c}8463 \\
(17.28 \%)\end{array}$ & $\begin{array}{c}2849 \\
(14.36 \%)\end{array}$ & $\begin{array}{c}5614 \\
(19.26 \%)\end{array}$ & $\begin{array}{c}2333 \\
(15.12 \%)\end{array}$ & $\begin{array}{c}4104 \\
(17.56 \%)\end{array}$ & $\begin{array}{c}2026 \\
(19.91 \%)\end{array}$ \\
\hline $\begin{array}{l}\text { The feeling } \\
\text { of suffering } \\
\text { from } \\
\text { undiagnosed } \\
\text { diseases }\end{array}$ & $\begin{array}{c}7253 \\
(14.81 \%)\end{array}$ & $\begin{array}{c}2580 \\
(13.01 \%)\end{array}$ & $\begin{array}{c}4673 \\
(16.03 \%)\end{array}$ & $\begin{array}{c}2009 \\
(13.02 \%)\end{array}$ & $\begin{array}{c}3743 \\
(16.01 \%)\end{array}$ & $\begin{array}{c}1501 \\
(14.75 \%)\end{array}$ \\
\hline
\end{tabular}


Compared with respondents without a family history of cardiovascular or cerebrovascular diseases, respondents with a family history of disease had a higher prevalence rate of suboptimal health status $(79.51 \%$ vs. $67.89 \%)$. Compared with respondents with normal blood pressure, hypertensive respondents had a higher prevalence rate of suboptimal health status $(71.83 \%$ vs. $68.45 \%)$. We observed higher prevalence rates of suboptimal health status among respondents with short sleep duration $(82.73 \%$ vs. $67.42 \%)$ and poor sleep quality $(72.64 \%$ vs. $61.41 \%)$ compared with their counterparts. Respondents who reported experiencing stress had a higher prevalence rate of suboptimal health status than their counterparts without stress $(86.96 \%$ vs. $69.18 \%)$, as did those who had experienced negative life events $(79.71 \%$ vs. $69.09 \%)$ or positive life events $(78.02 \%$ vs. $69.38 \%)$. Respondents who had unhealthy diet choices had a higher prevalence rate of suboptimal health status than their counterparts $(70.73 \%$ vs. $66.80 \%)$. Respondents who exercised regularly had a lower prevalence rate of suboptimal health status than those who did not exercise regularly (65.14\% vs. $73.13 \%)$, as did those who had regular meals at fixed hours ( $68.21 \%$ vs. $80.94 \%)$. There were no significant differences between different smoking conditions, drinking conditions, and BMI groups. The prevalence rates of suboptimal health status by different demographic characteristics and lifestyle factors are detailed in Table 2.

Table 3 presents the results of the bivariate and multivariate multi-level GEE models factors associated with suboptimal health status. After controlling for the cluster effect of living area, we found that gender, occupation, marital status, educational level, smoking, alcohol drinking, ethnicity, family history of cardiovascular or cerebrovascular diseases, sleep duration, sleep quality, stress, negative life events, exercise, diet choice, and meal times were associated with suboptimal health status. Tables 4 and 5 show the impact of independent variables on each dimension of suboptimal health separately based on multi-level GEE models. Independent associated factors were similar for six dimensions of suboptimal health, as shown in Tables 4 and 5.

Table 2. Prevalence (\%) of suboptimal health by respondents' demographic characteristics.

\begin{tabular}{|c|c|c|c|c|c|}
\hline \multirow{2}{*}{ Characteristics } & \multirow{2}{*}{ Total } & \multicolumn{2}{|c|}{ Sub-Health } & \multicolumn{2}{|c|}{ Chi-Square Test } \\
\hline & & Yes & No & Chi-Square & $p^{*}$ \\
\hline All Subjects & 48,978 & $34,021(59.46 \%)$ & 14,957 (30.54\%) & & \\
\hline Age (years) & & & & 215.8919 & $<0.0001$ \\
\hline $18-34$ & 15,430 & $10,033(65.02 \%)$ & 5397 (34.98\%) & & \\
\hline $35-59$ & 23,372 & $16,612(71.08 \%)$ & $6760(28.92 \%)$ & & \\
\hline$\geq 60$ & 10,176 & $7376(72.48 \%)$ & $2800(27.52 \%)$ & & \\
\hline Gender & & & & 349.9880 & $<0.0001$ \\
\hline Male & 19,834 & $12,841(64.74 \%)$ & $6993(35.26 \%)$ & & \\
\hline Female & 29,144 & $21,180(72.67 \%)$ & $7964(27.33 \%)$ & & \\
\hline Occupation & & & & 474.5250 & $<0.0001$ \\
\hline Blue-collar worker & 26,101 & $19,238(73.71 \%)$ & $6863(26.29 \%)$ & & \\
\hline White-collar worker & 22,877 & $14,783(64.62 \%)$ & $8094(35.38 \%)$ & & \\
\hline Marriage status & & & & 288.7890 & $<0.0001$ \\
\hline Married & 36,337 & $25,672(70.65 \%)$ & $10,665(29.35 \%)$ & & \\
\hline Single & 9972 & $6291(63.09 \%)$ & $3681(36.91 \%)$ & & \\
\hline Widowed or divorced & 2669 & $2058(77.11 \%)$ & $611(22.89 \%)$ & & \\
\hline Education level & & & & 404.9562 & $<0.0001$ \\
\hline Primary school & 10,881 & 7709 (76.47\%) & $2372(23.53 \%)$ & & \\
\hline Middle school & 22,597 & $15,758(69.73 \%)$ & $6839(30.27 \%)$ & & \\
\hline College & 16,300 & $10,554(64.75 \%)$ & $5746(35.25 \%)$ & & \\
\hline Smoker & & & & 1.8027 & 0.1794 \\
\hline No & 37,966 & $26,429(69.61 \%)$ & $11,537(30.39 \%)$ & & \\
\hline Yes & 11,012 & $7592(68.94 \%)$ & $3420(31.06 \%)$ & & \\
\hline Alcohol drinker & & & & 2.5979 & 0.1070 \\
\hline No & 38,065 & 26,509 (69.64\%) & $11,556(30.36 \%)$ & & \\
\hline Yes & 10,913 & $7512(68.84 \%)$ & $3401(31.16 \%)$ & & \\
\hline
\end{tabular}


Table 2. Cont

\begin{tabular}{|c|c|c|c|c|c|}
\hline \multirow{2}{*}{ Characteristics } & \multirow{2}{*}{ Total } & \multicolumn{2}{|c|}{ Sub-Health } & \multicolumn{2}{|c|}{ Chi-Square Test } \\
\hline & & Yes & No & Chi-Square & $p^{*}$ \\
\hline Ethnicity & & & & 436.9001 & $<0.0001$ \\
\hline Han & 36,663 & $25,242(68.85 \%)$ & $11,421(31.15 \%)$ & & \\
\hline$Y_{i}$ & 2451 & $2136(87.15 \%)$ & $315(12.85 \%)$ & & \\
\hline Miao & 675 & $424(62.81 \%)$ & $251(37.19 \%)$ & & \\
\hline Mongolia & 1802 & $1260(69.92 \%)$ & $542(30.08 \%)$ & & \\
\hline Tibetan & 896 & $600(66.96 \%)$ & $296(33.04 \%)$ & & \\
\hline Korean & 1574 & $1089(69.19 \%)$ & $485(30.81 \%)$ & & \\
\hline Hui & 2722 & $1865(68.52 \%)$ & $857(31.48 \%)$ & & \\
\hline Tujia & 1259 & $758(60.21 \%)$ & $501(39.79 \%)$ & & \\
\hline Others & 936 & $647(69.12 \%)$ & $289(30.88 \%)$ & & \\
\hline Family history & & & & 364.8801 & $<0.0001$ \\
\hline No & 42,354 & $28,754(67.89 \%)$ & $13,600(32.11 \%)$ & & \\
\hline Yes & 6624 & $5267(79.51 \%)$ & $1357(20.49 \%)$ & & \\
\hline BMI & & & & 5.7840 & 0.0555 \\
\hline Normal & 27,817 & $19,224(69.11 \%)$ & 8593 (30.89\%) & & \\
\hline Overweight & 15,284 & $10,645(69.65 \%)$ & $4639(30.35 \%)$ & & \\
\hline Obesity & 5877 & $4152(70.65 \%)$ & $1725(29.35 \%)$ & & \\
\hline Sleep duration & & & & 624.2102 & $<0.0001$ \\
\hline$\geq 6 \mathrm{~h}$ & 42,462 & $28,630(67.42 \%)$ & $13,832(32.58 \%)$ & & \\
\hline$<6 \mathrm{~h}$ & 6516 & $5391(82.73 \%)$ & $1125(17.27 \%)$ & & \\
\hline Sleep quality & & & & 591.6443 & $<0.0001$ \\
\hline Good & 13,874 & $8520(61.41 \%)$ & 5354 (38.59\%) & & \\
\hline Poor & 35,104 & $25,501(72.64 \%)$ & $9603(27.36 \%)$ & & \\
\hline Stress & & & & 114.6625 & $<0.0001$ \\
\hline No & 48,196 & $33,341(69.18 \%)$ & $14,855(30.85 \%)$ & & \\
\hline Yes & 782 & $680(86.96 \%)$ & $102(13.04 \%)$ & & \\
\hline Negative life event & & & & 89.0887 & $<0.0001$ \\
\hline No & 47,243 & $32,638(69.09 \%)$ & $14,605(30.91 \%)$ & & \\
\hline Yes & 1735 & $1383(79.71 \%)$ & $352(20.29 \%)$ & & \\
\hline Positive life event & & & & 15.8650 & $<0.0001$ \\
\hline No & 48,523 & $33,666(69.38 \%)$ & $14,857(30.62 \%)$ & & \\
\hline Yes & 455 & $355(78.02 \%)$ & $100(21.98 \%)$ & & \\
\hline Hypertension & & & & 55.3106 & $<0.0001$ \\
\hline No & 34,287 & $23,469(68.45 \%)$ & $10,818(31.55 \%)$ & & \\
\hline Yes & 14,691 & $10,552(71.83 \%)$ & $4139(28.17 \%)$ & & \\
\hline Regular exercise & & & & 365.9445 & $<0.0001$ \\
\hline No & 26,513 & $19,388(73.13 \%)$ & 7125 (26.87\%) & & \\
\hline Yes & 22,465 & $14,633(65.14 \%)$ & $7832(34.86 \%)$ & & \\
\hline Diet choice & & & & 78.194 & $<0.0001$ \\
\hline Routine & 15,834 & $10,577(66.80 \%)$ & $5257(33.20 \%)$ & & \\
\hline Unhealthy & 33,144 & $23,444(70.73 \%)$ & $9700(29.27 \%)$ & & \\
\hline Meal time & & & & 331.9804 & $<0.0001$ \\
\hline Regular & 44,157 & $30,119(68.21 \%)$ & $14,038(31.79 \%)$ & & \\
\hline Irregular & 4821 & $3902(80.94 \%)$ & $919(19.06 \%)$ & & \\
\hline
\end{tabular}

* Family history: family history of cardiovascular or cerebrovascular diseases. 
Table 3. Risk factors associated with suboptimal health based on a multi-level GEE model.

\begin{tabular}{|c|c|c|c|c|}
\hline \multirow{2}{*}{ Characteristics } & \multicolumn{2}{|c|}{ Bivariate } & \multicolumn{2}{|r|}{ Multivariate } \\
\hline & OR & $95 \%$ CI & OR & $95 \% \mathrm{CI}$ \\
\hline \multicolumn{5}{|l|}{ Age (years) } \\
\hline $18-34$ & 1.000 & - & 1.000 & - \\
\hline $35-59$ & 1.322 & $1.266-1.381$ & 1.040 & $0.977-1.108$ \\
\hline$\geq 60$ & 1.417 & $1.342-1.497$ & 1.067 & $0.979-1.163$ \\
\hline \multicolumn{5}{|l|}{ Gender } \\
\hline Male & 1.000 & - & 1.000 & - \\
\hline Female & 1.448 & $1.393-1.506$ & 1.284 & $1.198-1.377$ \\
\hline \multicolumn{5}{|l|}{ Occupation } \\
\hline Blue-collar worker & 1.535 & $1.477-1.595$ & 1.059 & $1.011-1.109$ \\
\hline White-collar worker & 1.000 & - & 1.000 & - \\
\hline \multicolumn{5}{|l|}{ Marriage status } \\
\hline Married & 1.000 & - & 1.000 & - \\
\hline Single & 0.710 & $0.678-0.744$ & 1.021 & $0.936-1.113$ \\
\hline Widowed or divorced & 1.399 & $1.276-1.537$ & 1.106 & $1.064-1.149$ \\
\hline \multicolumn{5}{|l|}{ Education level } \\
\hline Primary school & 1.000 & - & 1.000 & - \\
\hline Middle school & 0.709 & $0.672-0.748$ & 0.856 & $0.812-0.903$ \\
\hline College & 0.565 & $0.534-0.598$ & 0.791 & $0.730-0.856$ \\
\hline \multicolumn{5}{|l|}{ Smoker } \\
\hline No & 1.000 & - & 1.000 & - \\
\hline Yes & 0.969 & $0.926-1.015$ & 1.083 & $1.055-1.111$ \\
\hline \multicolumn{5}{|l|}{ Alcohol drinker } \\
\hline No & 1.000 & - & 1.000 & - \\
\hline Yes & 0.963 & $0.920-1.008$ & 1.075 & $1.025-1.127$ \\
\hline \multicolumn{5}{|l|}{ Ethnicity } \\
\hline Han & 1.000 & - & 1.000 & - \\
\hline Yi & 3.068 & $2.725-3.466$ & 1.002 & $0.921-1.089$ \\
\hline Miao & 0.764 & $0.653-0.896$ & 0.927 & $0.874-0.983$ \\
\hline Mongolia & 1.052 & $0.949-1.167$ & 0.824 & $0.801-0.847$ \\
\hline Tibetan & 0.917 & $0.797-1.057$ & 0.852 & $0.816-0.890$ \\
\hline Korean & 1.016 & $0.911-1.134$ & 0.981 & $0.929-1.036$ \\
\hline Hui & 0.985 & $0.906-1.071$ & 1.054 & $1.003-1.108$ \\
\hline Tujia & 0.685 & $0.610-0.768$ & 1.029 & $0.950-1.114$ \\
\hline Others & 1.013 & $0.881-1.167$ & 0.966 & $0.915-1.020$ \\
\hline \multicolumn{5}{|l|}{ Family history } \\
\hline No & 1.000 & - & 1.000 & - \\
\hline Yes & 1.836 & $1.724-1.956$ & 1.203 & $1.152-1.256$ \\
\hline BMI & & & & \\
\hline Normal & 1.000 & - & 1.000 & - \\
\hline Overweight & 1.026 & $0.983-1.071$ & 0.981 & $0.948-1.014$ \\
\hline Obesity & 1.076 & $1.012-1.144$ & 0.998 & $0.965-1.032$ \\
\hline Sleep duration & & & & \\
\hline$\geq 6 \mathrm{~h}$ & 1.000 & - & 1.000 & - \\
\hline$<6 \mathrm{~h}$ & 2.315 & $2.165-2.478$ & 1.235 & $1.183-1.290$ \\
\hline Sleep quality & & & & \\
\hline Good & 1.000 & - & 1.000 & - \\
\hline Poor & 1.669 & $1.601-1.739$ & 1.594 & $1.516-1.677$ \\
\hline Stress & & & & \\
\hline No & 1.000 & - & 1.000 & - \\
\hline Yes & 2.970 & $2.422-3.681$ & 1.588 & $1.496-1.686$ \\
\hline Negative life event & & & & \\
\hline No & 1.000 & - & 1.000 & - \\
\hline Yes & 1.758 & $1.563-1.982$ & 1.144 & $1.045-1.187$ \\
\hline Positive life event & & & & \\
\hline No & 1.000 & - & 1.000 & - \\
\hline Yes & 1.566 & $1.259-1.967$ & 1.014 & $0.973-1.056$ \\
\hline Hypertension & & & & \\
\hline No & 1.000 & - & 1.000 & - \\
\hline Yes & 1.175 & $1.126-1.226$ & 1.000 & $0.977-1.022$ \\
\hline Regular exercise & & & & \\
\hline No & 1.000 & - & 1.000 & - \\
\hline Yes & 0.687 & $0.661-0.714$ & 0.913 & $0.849-0.983$ \\
\hline Diet choice & & & & \\
\hline Routine & 1.000 & - & 1.000 & - \\
\hline Unhealthy & 1.201 & $1.153-1.251$ & 1.093 & $1.033-1.156$ \\
\hline Meal time & & & & \\
\hline Regular & 1.000 & - & 1.000 & - \\
\hline Irregular & 1.979 & $1.838-2.133$ & 1.231 & $1.105-1.372$ \\
\hline
\end{tabular}

OR: odds ratio; CI: confidence interval; GEE: generalized estimating equation; Family history: family history of cardiovascular or cerebrovascular diseases. 
Table 4. Risk factors associated with each dimension of suboptimal health based on a multi-level multivariate GEE model.

\begin{tabular}{|c|c|c|c|c|c|c|}
\hline \multirow{2}{*}{ Characteristics } & \multicolumn{2}{|c|}{ Physical Symptom } & \multicolumn{2}{|c|}{ Psychological Symptom } & \multicolumn{2}{|c|}{ Vigor } \\
\hline & OR & $95 \%$ CI & OR & $95 \% \mathrm{CI}$ & OR & $95 \%$ CI \\
\hline \multicolumn{7}{|l|}{ Age (years) } \\
\hline $18-34$ & 1.000 & - & 1.000 & - & 1.000 & - \\
\hline $35-59$ & 1.119 & $1.055-1.185$ & 1.000 & $0.938-1.065$ & 1.004 & $0.917-1.099$ \\
\hline$\geq 60$ & 1.143 & $1.055-1.238$ & 0.996 & $0.912-1.088$ & 1.170 & $1.023-1.339$ \\
\hline \multicolumn{7}{|l|}{ Gender } \\
\hline Male & 1.000 & - & 1.000 & - & 1.000 & - \\
\hline Female & 1.260 & $1.175-1.353$ & 1.336 & $1.243-1.436$ & 1.246 & $1.166-1.331$ \\
\hline \multicolumn{7}{|l|}{ Occupation } \\
\hline Blue-collar worker & 1.086 & $1.040-1.133$ & 1.047 & $0.998-1.098$ & 1.032 & $0.956-1.114$ \\
\hline White-collar worker & 1.000 & - & 1.000 & - & 1.000 & - \\
\hline \multicolumn{7}{|l|}{ Marriage status } \\
\hline Married & 1.000 & - & 1.000 & - & 1.000 & - \\
\hline ingle & 0.790 & $0.696-0.897$ & 1.156 & $1.065-1.256$ & 1.167 & $1.045-1.304$ \\
\hline Widowed/divorced & 1.056 & $1.021-1.093$ & 1.162 & $1.107-1.220$ & 1.165 & $1.101-1.232$ \\
\hline \multicolumn{7}{|l|}{ Education level } \\
\hline Primary school & 1.000 & - & 1.000 & - & 1.000 & - \\
\hline Middle school & 0.836 & $0.789-0.887$ & 0.913 & $0.866-0.962$ & 0.870 & $0.803-0.941$ \\
\hline College & 0.746 & $0.681-0.818$ & 0.855 & $0.790-0.924$ & 0.855 & $0.778-0.940$ \\
\hline \multicolumn{7}{|l|}{ Smoker } \\
\hline No & 1.000 & - & 1.000 & - & 1.000 & - \\
\hline Yes & 1.125 & $1.086-1.165$ & 1.038 & $1.011-1.066$ & 1.072 & $1.024-1.121$ \\
\hline \multicolumn{7}{|l|}{ Alcohol drinker } \\
\hline No & 1.000 & - & 1.000 & - & 1.000 & - \\
\hline Yes & 1.044 & $1.006-1.083$ & 1.095 & $1.040-1.153$ & 1.111 & $1.027-1.202$ \\
\hline Ethnicity & & & & & & \\
\hline Han & 1.000 & - & 1.000 & - & 1.000 & - \\
\hline Yi & 1.003 & $0.873-1.152$ & 1.039 & $0.949-1.138$ & 0.901 & $0.832-0.976$ \\
\hline Miao & 0.922 & $0.878-0.968$ & 0.946 & $0.874-1.025$ & 0.880 & $0.814-0.952$ \\
\hline Mongolia & 0.904 & $0.881-0.927$ & 0.781 & $0.744-0.820$ & 0.703 & $0.626-0.790$ \\
\hline Tibetan & 0.944 & $0.891-1.000$ & 0.722 & $0.683-0.763$ & 0.731 & $0.665-0.804$ \\
\hline Korean & 0.986 & $0.927-1.050$ & 0.915 & $0.876-0.957$ & 0.950 & $0.829-1.089$ \\
\hline Hui & 1.066 & $0.999-1.137$ & 1.046 & $0.997-1.097$ & 1.046 & $0.983-1.112$ \\
\hline Tujia & 1.015 & $0.978-1.054$ & 1.039 & $0.942-1.147$ & 1.010 & $0.891-1.145$ \\
\hline Others & 0.910 & $0.820-1.011$ & 0.986 & $0.942-1.147$ & 1.014 & $0.971-1.059$ \\
\hline Disease family history & & & & & & \\
\hline No & 1.000 & - & 1.000 & - & 1.000 & - \\
\hline Yes & 1.196 & $1.141-1.255$ & 1.196 & $1.152-1.242$ & 1.202 & $1.142-1.265$ \\
\hline BMI & & & & & & \\
\hline Normal & 1.000 & - & 1.000 & - & 1.000 & - \\
\hline Overweight & 1.001 & $0.965-1.038$ & 0.976 & $0.942-1.011$ & 0.973 & $0.928-1.021$ \\
\hline Obesity & 1.041 & $1.003-1.080$ & 0.985 & $0.950-1.020$ & 0.969 & $0.917-1.025$ \\
\hline Sleep duration & & & & & & \\
\hline$\geq 6 \mathrm{~h}$ & 1.000 & - & 1.000 & - & 1.000 & - \\
\hline$<6 \mathrm{~h}$ & 1.232 & $1.180-1.286$ & 1.268 & $1.222-1.315$ & 1.204 & $1.150-1.260$ \\
\hline Sleep quality & & & & & & \\
\hline Good & 1.000 & - & 1.000 & - & 1.000 & - \\
\hline Poor & 1.398 & $1.332-1.468$ & 1.814 & $1.696-1.941$ & 1.556 & $1.473-1.643$ \\
\hline Stress & & & & & & \\
\hline No & 1.000 & - & 1.000 & - & 1.000 & - \\
\hline Yes & 1.400 & $1.327-1.477$ & 1.558 & $1.411-1.721$ & 2.033 & $1.830-2.258$ \\
\hline Negative life event & & & & & & \\
\hline No & 1.000 & - & 1.000 & - & 1.000 & - \\
\hline Yes & 1.048 & $0.912-1.206$ & 1.130 & $1.102-1.158$ & 1.180 & $1.091-1.276$ \\
\hline Positive life event & & & & & & \\
\hline No & 1.000 & - & 1.000 & - & 1.000 & - \\
\hline Yes & 0.904 & $0.826-0.989$ & 1.069 & $1.024-1.114$ & 1.109 & $1.024-1.202$ \\
\hline Hypertension & & & & & & \\
\hline No & 1.000 & - & 1.000 & - & 1.000 & - \\
\hline Yes & 1.014 & $0.994-1.034$ & 0.986 & $0.966-1.006$ & 1.014 & $0.971-1.059$ \\
\hline Regular exercise & & & & & & \\
\hline No & 1.000 & - & 1.000 & - & 1.000 & - \\
\hline Yes & 0.945 & $0.879-1.015$ & 0.925 & $0.867-0.987$ & 0.824 & $0.746-0.910$ \\
\hline Diet choice & & & & & & \\
\hline Routine & 1.000 & - & 1.000 & - & 1.000 & - \\
\hline Unhealthy & 1.062 & $1.001-1.127$ & 1.107 & $1.050-1.167$ & 1.145 & $1.064-1.232$ \\
\hline Meal time & & & & & & \\
\hline Regular & 1.000 & - & 1.000 & - & 1.000 & - \\
\hline Irregular & 1.130 & $0.896-1.426$ & 1.256 & $1.193-1.322$ & 1.317 & $1.262-1.375$ \\
\hline
\end{tabular}

OR: odds ratio; CI: confidence interval; GEE: generalized estimating equation; Family history: family history of cardiovascular or cerebrovascular diseases. The physical symptoms dimension contains five items: fatigue, headache or dizziness, tinnitus, numbness or stiffness in the shoulders or legs, and a sense of pharyngeal foreign bodies. The psychological symptoms dimension contains six items: upset, loneliness, inattention, anxiety, dreaminess, and forgetfulness. The vigor dimension contains three items: decreased vitality, disinterest in surroundings, and moodiness. 
Table 5. Risk factors associated with each dimension of suboptimal health based on a multi-level multivariate GEE model.

\begin{tabular}{|c|c|c|c|c|c|c|}
\hline \multirow{2}{*}{ Characteristics } & \multicolumn{2}{|c|}{ Social Adaptability } & \multicolumn{2}{|c|}{ Immunity } & \multicolumn{2}{|c|}{ Immunity andGoing to Hospital } \\
\hline & OR & $95 \% \mathrm{CI}$ & OR & $95 \% \mathrm{CI}$ & OR & $95 \% \mathrm{CI}$ \\
\hline \multicolumn{7}{|l|}{ Age (years) } \\
\hline $18-34$ & 1.000 & - & 1.000 & - & 1.000 & - \\
\hline $35-59$ & 0.950 & $0.863-1.046$ & 0.978 & $0.902-1.060$ & 1.076 & $0.969-1.194$ \\
\hline$\geq 60$ & 0.757 & $0.635-0.903$ & 1.120 & $1.022-1.227$ & 1.031 & $0.896-1.186$ \\
\hline \multicolumn{7}{|l|}{ Gender } \\
\hline Male & 1.000 & - & 1.000 & - & 1.000 & - \\
\hline Female & 1.099 & $1.003-1.205$ & 1.306 & $1.216-1.403$ & 1.276 & $1.103-1.477$ \\
\hline \multicolumn{7}{|l|}{ Occupation } \\
\hline Blue-collar worker & 0.943 & $0.842-1.055$ & 1.146 & $1.081-1.215$ & 1.119 & $1.038-1.206$ \\
\hline White-collar worker & 1.000 & - & 1.000 & - & 1.000 & - \\
\hline \multicolumn{7}{|l|}{ Marriage status } \\
\hline Married & 1.000 & - & 1.000 & - & 1.000 & - \\
\hline Single & 1.125 & $0.972-1.303$ & 0.939 & $0.786-1.123$ & 0.947 & $0.826-1.087$ \\
\hline Widowed/divorced & 1.115 & $1.035-1.202$ & 0.989 & $0.911-1.074$ & 0.995 & $0.894-1.107$ \\
\hline \multicolumn{7}{|l|}{ Education level } \\
\hline Primary school & 1.000 & - & 1.000 & - & 1.000 & - \\
\hline Middle school & 0.774 & $0.706-0.849$ & 0.740 & $0.661-0.828$ & 0.756 & $0.660-0.866$ \\
\hline College & 0.622 & $0.553-0.700$ & 0.655 & $0.544-0.790$ & 0.764 & $0.673-0.867$ \\
\hline \multicolumn{7}{|l|}{ Smoker } \\
\hline No & 1.000 & - & 1.000 & - & 1.000 & - \\
\hline Yes & 1.141 & $1.060-1.228$ & 1.113 & $1.046-1.185$ & 1.089 & $1.039-1.142$ \\
\hline \multicolumn{7}{|l|}{ Alcohol drinker } \\
\hline No & 1.000 & - & 1.000 & - & 1.000 & - \\
\hline Yes & 1.034 & $0.950-1.125$ & 1.002 & $0.944-1.065$ & 1.140 & $1.023-1.270$ \\
\hline Ethnicity & & & & & & \\
\hline Han & 1.000 & - & 1.000 & - & 1.000 & - \\
\hline $\mathrm{Yi}$ & 0.898 & $0.702-1.149$ & 1.095 & $0.654-1.833$ & 1.413 & $1.003-1.992$ \\
\hline Miao & 0.991 & $0.790-1.243$ & 0.963 & $0.793-1.170$ & 0.835 & $0.732-0.953$ \\
\hline Mongolia & 0.835 & $0.668-1.042$ & 0.956 & $0.902-1.013$ & 0.931 & $0.823-1.053$ \\
\hline Tibetan & 0.789 & $0.626-0.995$ & 1.258 & $1.094-1.446$ & 1.187 & $1.042-1.351$ \\
\hline Korean & 1.280 & $1.046-1.566$ & 1.224 & $1.089-1.376$ & 1.100 & $0.960-1.261$ \\
\hline Hui & 0.985 & $0.855-1.135$ & 1.056 & $0.977-1.141$ & 1.121 & $1.040-1.351$ \\
\hline Tujia & 0.868 & $0.722-1.042$ & 1.001 & $0.807-1.241$ & 1.278 & $1.148-1.424$ \\
\hline Others & 1.035 & $0.885-1.210$ & 0.860 & $0.676-1.094$ & 1.079 & $0.899-1.293$ \\
\hline Disease family history & & & & & & \\
\hline No & 1.000 & - & 1.000 & - & 1.000 & - \\
\hline Yes & 1.200 & $1.060-1.357$ & 1.212 & $1.118-1.315$ & 1.308 & $1.229-1.392$ \\
\hline BMI & & & & & & \\
\hline Normal & 1.000 & - & 1.000 & - & 1.000 & - \\
\hline Overweight & 0.976 & $0.919-1.038$ & 0.929 & $0.880-0.980$ & 0.946 & $0.900-0.993$ \\
\hline Obesity & 0.948 & $0.850-1.058$ & 0.926 & $0.860-0.997$ & 0.966 & $0.902-1.033$ \\
\hline Seep duration & & & & & & \\
\hline$\geq 6 \mathrm{~h}$ & 1.000 & - & 1.000 & - & 1.000 & - \\
\hline$<6 \mathrm{~h}$ & 1.194 & $1.068-1.335$ & 1.215 & $1.094-1.350$ & 1.158 & $0.986-1.359$ \\
\hline Sleep quality & & & & & & \\
\hline Good & 1.000 & - & 1.000 & - & 1.000 & - \\
\hline Poor & 1.534 & $1.357-1.733$ & 1.507 & $1.377-1.650$ & 1.774 & $1.485-2.120$ \\
\hline Stress & & & & & & \\
\hline No & 1.000 & - & 1.000 & - & 1.000 & - \\
\hline Yes & 2.346 & $2.054-2.681$ & 1.284 & $1.240-1329$ & 1.459 & $1.378-1.544$ \\
\hline Negative life event & & & & & & \\
\hline No & 1.000 & - & 1.000 & - & 1.000 & - \\
\hline Yes & 1.277 & $1.144-1.425$ & 1.104 & $0.885-1.376$ & 1.197 & $0.917-1.563$ \\
\hline Positive life event & & & & & & \\
\hline No & 1.000 & - & 1.000 & - & 1.000 & - \\
\hline Yes & 1.257 & $1.133-1.394$ & 0.987 & $0.726-1.342$ & 0.779 & $0.695-0.874$ \\
\hline Hypertension & & & & & & \\
\hline No & 1.000 & - & 1.000 & - & 1.000 & - \\
\hline Yes & 0.949 & $0.887-1.016$ & 1.016 & $0.977-1.057$ & 0.978 & $0.932-1.026$ \\
\hline Regular exercise & & & & & & \\
\hline No & 1.000 & - & 1.000 & - & 1.000 & - \\
\hline Yes & 0.873 & $0.720-1.060$ & 0.910 & $0.825-1.004$ & 0.897 & $0.766-1.049$ \\
\hline Diet choice & & & & & & \\
\hline Routine & 1.000 & - & 1.000 & - & 1.000 & - \\
\hline Unhealthy & 1.037 & $0.927-1.160$ & 1.107 & $1.003-1.221$ & 1.069 & $0.962-1.187$ \\
\hline Meal time & & & & & & \\
\hline Regular & 1.000 & - & 1.000 & - & 1.000 & - \\
\hline Irregular & 1.505 & $1.404-1.612$ & 1.168 & $1.062-1.284$ & 1.299 & $1.118-1.509$ \\
\hline
\end{tabular}

OR: odds ratio; CI: confidence interval; GEE: generalized estimating equation; Family history: family history of cardiovascular or cerebrovascular diseases. The social adaptability dimension contains two items: feeling tired at work and incompatibility with coworkers. The immunity dimension contains one item: susceptibility to flu or other diseases. The going to hospital dimension contains one item: the feeling of suffering from undiagnosed diseases. 


\section{Discussion}

In multi-stage sampling, survey data have a hierarchical structure in which individuals are nested within higher level sampling units. Hierarchical social structures naturally generate multi-level data in which the lower level units are nested in the next higher level units. Individual suboptimal health status is determined by individual characteristics (e.g., age, ethnicity, education, and occupation at the micro level) as well as features of the social contexts or social environments in which individuals live (e.g., culture or subculture). Factors such as diet choice/preferences at the macro level can also produce measurable effects. People living in the same neighborhood may share similarities because they are influenced by the same neighborhood socioeconomic characteristics. In statistical terms, there is within-group homogeneity and between-group heterogeneity in hierarchically structured data [23]. Traditional analytical methods such as logistic regression assume that observations are independently but identically distributed. Violation of this assumption results in incorrect inferences in statistical analyses. Multi-level GEE models provide an appropriate analytical framework to deal with observation dependence in multi-level data. More importantly, multi-level models permit exploration of the nature and extent of relationships at both micro and macro levels, as well as across levels [23]. We considered that individuals living in the same area were likely to suffer from the effects of similar living circumstances, which would have an inevitable confusing influence on the accuracy and generalization of the study results concerning suboptimal health status. This was the first study to explore the prevalence of suboptimal health status and the relationships of suboptimal health status and lifestyle factors using multi-level GEE models controlled for the cluster effect of the same living area and similar living circumstances to guarantee the accuracy and generalizability of the study results.

We found that more than two-thirds of Chinese adults had suboptimal health status. The prevalence of suboptimal health status in this study was similar to that in several previous reports $[6,7,24]$, but significantly higher than that in a report by Wang et al. $[25,26]$. Wang et al. reported that the prevalence of suboptimal health status was $9.0 \%$, based on the Suboptimal Health Status Questionnaire-25 (SHSQ-25) administered in a cross-sectional survey with 4313 participants [25]. The low prevalence reported by Wang et al. was mainly attributable to different participant selection criteria and a higher threshold for scores. Suboptimal health is often assessed with an assessment scale, such as the Delphi suboptimal health scale used in our study and the SHSQ-25 [25,26]. The SHSQ-25 contains 25 items under five domains (fatigue, the cardiovascular system, the digestive tract, the immune system, and mental status). Participants are asked to rate specific statements on a five-point scale, with raw scores of 1-5 recorded as $0-4$. Total scores are calculated for each participant by summing the ratings for the 25 items. The 25 SHSQ-25 items are similar as to the 18 symptoms covered in Delphi scale, although the SHSQ-25 items are more delicate. With the SHSQ-25, a high score $(\geq 35)$ represents poor health, and is regarded as suboptimal health status $[25,26]$. In other words, having more than one-third of the total possible score (100) in the SHSQ-25 was considered to reflect suboptimal health status. In our study, respondents were considered as having suboptimal health status if they suffered from more than one-third of the 18 symptoms ( $\geq 7$ symptoms); the prevalence of suboptimal health status was 19.24\%. In addition, Wang et al. [25] included 4313 participants aged 18-65 years, whereas participants currently suffering from diabetes, hypertension, hyperlipidemia, cardiovascular or cerebrovascular conditions, any type of cancer, and gout were excluded. In our study, $13.07 \%$ of respondents were aged over 65 years and we only excluded those who suffered from serious chronic diseases and had run a high fever in the past 15 days. Older participants and those with mild chronic diseases were also more likely to be classified as having suboptimal health status than other participants, which may explain the higher prevalence found in our study. If those who were aged over 65 years or currently suffering from mild chronic diseases were excluded, the prevalence of suboptimal health status might be similar to that in the previous report.

Unhealthy lifestyles were closely related to both chronic diseases and suboptimal health status. In a recent report, Ma et al. noted that good sleep quality, abundant physical exercise, and adequate nutrition intake were negatively associated with suboptimal health status, whereas overuse of electronic 
devices and weight loss were positively associated with suboptimal health status [24]. Chen et al. reported that poor stress management, poor self-actualization, lack of exercise, and poor interpersonal relationships were positively associated with suboptimal health status [15]. Suboptimal health has been observed to be related to low levels of physical activity and exercise, which are known risk factors for cardiovascular and cerebrovascular diseases [27]. Smoking and drinking alcohol have been confirmed as associated with many chronic diseases [28-31] and were also associated with suboptimal health status in our study. Sleep was defined as a natural and reversible state of reduced responsiveness to external stimuli and relative inactivity, accompanied by a loss of consciousness. Sleep problems and sleep continuity were significant predictors of relapse. Sleep deprivation and sleep disruptions may cause severe cognitive and emotional problems [32,33]. Previous studies reported that inadequate sleep duration caused heightened cardiovascular risk, cerebrovascular disease, psychiatric disorders, and obesity [34-37].

In addition to common unhealthy lifestyle factors, such as lack of exercise, smoking, drinking alcohol, short sleep duration, and poor sleep quality, we found that stress, negative life events, family history of cardiovascular or cerebrovascular diseases, unhealthy diet choices, and irregular meal times were associated with a higher prevalence risk of suboptimal health.

$\mathrm{Wu}$ et al. reported that poor work-recreation balance was associated with increased risk for suboptimal health status [38]. Chang et al. found that suboptimal health status among middle school teachers was closely related to occupational stress [39]. Negative life events and work, home, or economic stress were important factors for depression and anxiety, and may further lead to suboptimal health status. Respondents with parents that suffered from cardiovascular or cerebrovascular diseases may experience anxiety about suffering these diseases themselves, which may in turn make them more susceptible to suboptimal health status.

We found that both unhealthy diet choices and irregular meal times were closely related to a higher prevalence of suboptimal health. Owen et al. confirmed the role of diet and nutrition in mental health and wellbeing [40]. In addition, a high-salt diet was reported to be closely associated with hypertension [41]. Cordner et al. reported that the associations between consumption of a high-fat or a "Western" diet and metabolic disorders (e.g., obesity, diabetes, and cardiovascular disease) have long been recognized, and a large body of evidence suggests that diets high in fat could also have a profound impact on the brain, behavior, and cognition [42]. A large prospective study found the habitual consumption of spicy foods was inversely associated with total and certain cause specific mortality, independent of other risk factors for death [43]. Regularly having breakfast, lunch, and dinner at fixed times may induce individuals to work and live within a routine, which may help them to maintain healthy living habits and timetables, which could benefit their physical and psychological health and in turn help to prevent anxiety and suboptimal health status.

This study had several limitations that should be mentioned. First, because of the cross-sectional design, it was not possible to confirm causal relationships between suboptimal health and lifestyle factors. Second, suboptimal health has several different definitions, which limited the comparison of our study results with other reports on the prevalence of suboptimal health. Third, lifestyle factors were self-reported in this study, which reduced the precision of measurement of these factors, such as smoking, alcohol drinking, sleep habits, diet choices, and meal times. Finally, since the survey data referred to years 2007-2011 and the study findings reflected the situation in 2007-2011, they may not reflect the current situation.

\section{Conclusions}

Despite these limitations, our findings showed that suboptimal health has become a serious public health challenge in China. Unhealthy lifestyles were closely associated with suboptimal health, with key factors being smoking, drinking alcohol, short sleep duration, poor sleep quality, lack of regular exercise, stress, negative life events, family history of cardiovascular and cerebrovascular 
diseases, unhealthy diet choices, and irregular meal times. The health status of the population could be effectively improved by improving lifestyle behaviors.

Author Contributions: T.X. participated in the design of the study and the field survey, preformed the statistical analysis, and drafted the manuscript. G.Z. conceived the study. S.H. conceived the study and participated in its design. All authors read and approved the final manuscript.

Funding: This work was supported by CAMS Innovation Fund for Medical Sciences (2018-I2M-AI-009) and the basic performance key project by the Ministry of Science and Technology of the People's Republic of China (No. 2006FY110300). And the APC was funded by CAMS Innovation Fund for Medical Sciences (2018-I2M-AI-009).

Acknowledgments: We wish to thank all respondents who generously gave their time to participate in this research. We also thank Audrey Holmes, MA, from Liwen Bianji, Edanz Group China (www.liwenbianji.cn/ac), for editing the English text of a draft of this manuscript.

Conflicts of Interest: The authors declare no conflict of interest.

\section{References}

1. Grad, F.P. The preamble of the constitution of the World Health Organization. Bull. World Health Organ. 2002, 80, 981-984. [PubMed]

2. Medicine CAOC. The TCM Clinical Guidelines of Suboptimal Health Status; China Press TCM: Beijing, China, 2006.

3. Wang, W.; Yan, Y. Suboptimal health: A new health dimension for translational medicine. Clin. Transl. Med. 2012, 1, 28. [CrossRef] [PubMed]

4. Ke, B.; Liang, Y. Anti-aging and complete sub-health checkup. Clin. Funct. Nutr. 2011, 3, 137-140.

5. Dunstan, R.H.; Sparkes, D.L.; Roberts, T.K.; Crompton, M.J.; Gottfries, J.; Dascombe, B.J. Development of a complex amino acid supplement, fatigue Reviva, for oral ingestion: Initial evaluations of product concept and impact on symptoms of sub-health in a group of males. Nutr. J. 2013, 12, 115. [CrossRef]

6. Wang, D.X.; Zhou, H.L. Healthy, disease and subhealthy. Med. Soc. 2007, 20, 5-8.

7. Xie, Y.M.; Liu, B.Y.; Piao, H.Y. Exploration on the common characters of sub-healthy people based on clinical epidemiology. J. Chin. Integr. Med. 2006, 26, 612-616.

8. Sun, T.; Wang, T.F.; Wu, L.X. Sub-Health; China Press of Traditional Chinese Medicine: Beijing, China, 2007; pp. 16-17.

9. Patel, S.R.; Hu, F.B. Short sleep duration and weight gain: A systematic review. Obesity 2008, 16, 643-653. [CrossRef]

10. Van Cauter, E.; Spiegel, K.; Tasali, E.; Leproult, R. Metabolic consequences of sleep and sleep loss. Sleep Med. 2008, 9 (Suppl. 1), S23-S28. [CrossRef]

11. Okami, Y.; Kato, T.; Nin, G.; Harada, K.; Aoi, W.; Wada, S.; Higashi, A.; Okuyama, Y.; Takakuwa, S.; Ichikawa, $\mathrm{H}$.; et al. Lifestyle and psychological factors related to irritable bowel syndrome in nursing and medical school students. J. Gastroenterol. 2011, 46, 1403-1410. [CrossRef]

12. Folsom, A.R.; Yatsuya, H.; Nettleton, J.A.; Lutsey, P.L.; Cushman, M.; Rosamond, W.D.; ARIC Study Investigators. Community prevalence of ideal cardiovascular health, by the American Heart Association definition, and relationship with cardiovascular disease incidence. J. Am. Coll. Cardiol. 2011, 57, 1690-1696. [CrossRef]

13. Lin, C.C.; Li, C.I.; Liu, C.S.; Lin, W.Y.; Fuh, M.M.T.; Yang, S.Y.; Lee, C.C.; Li, T.C. Impact of lifestyle-related factors on all-cause and cause-specific mortality in patients with type 2 diabetes the Taichung Diabetes Study. Diabetes Care 2012, 35, 105-112. [CrossRef] [PubMed]

14. Sikorski, C.; Luppa, M.; Weyerer, S.; König, H.-H.; Maier, W.; Schön, G.; Petersen, J.J.; Gensichen, J.; Fuchs, A.; Bickel, H.; et al. Obesity and associated lifestyle in a large sample of Multi- Morbid German primary care attendees. Plos One 2014, 9, e102587. [CrossRef] [PubMed]

15. Chen, J.; Xiang, H.; Jiang, P.; König, H.-H.; Maier, W.; Schön, G.; Petersen, J.J.; Gensichen, J.; Fuchs, A.; Bickel, H.; et al. The role of healthy lifestyle in the implementation of regressing suboptimal health status among college students in China: A nested case-control study. Int. J. Environ. Res. Public Health 2017, 14, 240. [CrossRef] [PubMed]

16. Sun, X.M.; Wei, M.; Zhu, C.Y. An investigation of suboptimal health status in Guangdong: A cross section study. Shandong Med. J. 2008, 48, 59-60. 
17. Chen, Q.S.; Wang, S.Y.; Jing, C.X.; Dong, X.M.; Chi, G.B. Evaluation on diagnostic criterion of suboptimal health with Delphi method. Chin. J. Public Health 2003, 19, 1467-1468.

18. Xu, T.; Han, S.M.; Liu, J.T.; Zhu, G.J.; Mao, M. Comparison of suboptimal health status between Tibetan people and Han people. Chin. Med. J. 2009, 89, 2671-2674.

19. Xu, T.; Liu, J.T.; Han, S.M.; Zhu, G.J.; Mao, M. Analyses for Risk Factors of suboptimal health status with Logistic Model and Binomial Model—Survey in Tibetan People. J. Clin. Rehabil. Tissue Eng. Res. 2009, 13, 6597-6600.

20. Zhou, B.F. Effect of body mass index on all-cause mortality and incidence of cardiovascular diseases-report for meta-analysis of prospective studies open optimal cut-off points of body mass index in Chinese adults. Biomed. Environ. Sci. 2002, 15, 245-252.

21. Chinese Guidelines for Hypertension Prevention and Treatment Revision Committee. Chinese Guidelines for Hypertension Prevention and Treatment (Revised Edition 2005); People's Medical Publishing House: Beijing, China, 2006.

22. National Institutes of Health; National Heart, Lung, and Blood Institute. National High Blood Pressure Education Program; National Institutes of Health: Bethesda, MD, USA, 2003.

23. Wang, J.; Xie, H.; Fisher, J.H. Multilevel Models: Applications Using SAS; Higher Education Press: Beijing, China, 2010.

24. Ma, C.; Xu, W.; Zhou, L.; Ma, S.; Wang, Y. Association between lifestyle factors and suboptimal health status among Chinese college freshmen: A cross-sectional study. BMC Public Health 2018, 18, 105. [CrossRef]

25. Yan, Y.; Liu, Y.; Li, M.; Hu, P.-F.; Guo, A.-M.; Yang, X.-H.; Qiu, J.-J.; Yang, S.-S.; Shen, J.; Zhang, L.-P.; et al. Development and evaluation of a questionnaire for measuring suboptimal health status in urban Chinese. J. Epidemiol. 2009, 19, 333-341. [CrossRef]

26. Wang, Y.; Ge, S.; Yan, Y.; Wang, A.; Zhao, Z.; Yu, X.; Qiu, J.; AlZain, M.A.; Wang, H.; Fang, H.; et al. China suboptimal health cohort study: Rationale, design and baseline characteristics. J. Transl. Med. 2016, 14, 291. [CrossRef] [PubMed]

27. Ambrose, J.A.; Barua, R.S. The pathophysiology of cigarette smoking and cardiovascular disease: An update. J. Am. Coll. Cardiol. 2004, 43, 1731-1737. [CrossRef] [PubMed]

28. Sasco, A.J.; Secretan, M.B.; Straif, K. Tobacco smoking and cancer: A brief review of recent epidemiological evidence. Lung Cancer 2004, 45 (Suppl. 2), S3-S9. [CrossRef] [PubMed]

29. Akter, S.; Goto, A.; Mizoue, T. Smoking and the risk of type 2 diabetes in Japan: A systematic review and meta-analysis. J. Epidemiol. 2017, 27, 553-561. [CrossRef]

30. Erol, A.; Karpyak, V.M. Sex and gender-related differences in alcohol use and its consequences: Contemporary knowledge and future research considerations. Drug Alcohol Depend. 2015, 156, 1-13. [CrossRef]

31. Cohen, S.M. Alcoholic Liver Disease. Clin. Liver Dis. 2016, 20, xiii-xiv. [CrossRef]

32. Brown, L.K. Can sleep deprivation studies explain why human adults sleep? Curr. Opin. Pulm. Med. 2012, 18, 541-545. [CrossRef]

33. Killgore, W.D.S. Effects of sleep deprivation on cognition. Prog. Brain Res. 2010, 185, 105-129.

34. Covassin, N.; Singh, P. Sleep Duration and Cardiovascular Disease Risk: Epidemiologic and Experimental Evidence. Sleep Med Clin. 2016, 11, 81-89. [CrossRef]

35. Ge, B.H.; Guo, X.M. Short and long sleep durations are both associated with increased risk of stroke: A meta-analysis of observational studies. Int. J. Stroke 2015, 10, 177-184. [CrossRef]

36. Ranum, B.M.; Wichstrøm, L.; Pallesen, S.; Falch-Madsen, J.; Halse, M.; Steinsbekk, S. Association Between Objectively Measured Sleep Duration and Symptoms of Psychiatric Disorders in Middle Childhood. JAMA Netw. Open 2019, 2, e1918281. [CrossRef] [PubMed]

37. Ning, X.; Lv, J.; Guo, Y.; Bian, Z.; Tan, Y.; Pei, P.; Chen, J.; Yan, S.; Li, H.; Fu, Z.; et al. Association of Sleep Duration with Weight Gain and General and Central Obesity Risk in Chinese Adults: A Prospective Study. Obesity 2019. [CrossRef] [PubMed]

38. Wu, S.; Xuan, Z.; Li, F.; Xiao, W.; Fu, X.; Jiang, P.; Chen, J.; Xiang, L.; Liu, Y.; Nie, X.; et al. Work-Recreation Balance, Health-Promoting Lifestyles and Suboptimal Health Status in Southern China: A Cross-Sectional Study. Int. J. Environ. Res. Public Health 2016, 13, E339. [CrossRef] [PubMed]

39. Chang, W.; Shao, H.; Zhi, X.; Xu, J.; Xie, J. Sub-health status of middle school teachers and its correlation analysis with occupational stress. Chin. J. Ind. Hyg. Occup. Dis. 2017, 35, 585-588. 
40. Owen, L.; Corfe, B. The role of diet and nutrition on mental health and wellbeing. Proc. Nutr. Soc. 2017, 76, 425-426. [CrossRef] [PubMed]

41. Garfinkle, M.A. Salt and essential hypertension: Pathophysiology and implications for treatment. J Am Soc Hypertens. 2017, 11, 385-391. [CrossRef]

42. Cordner, Z.A.; Tamashiro, K.L. Effects of high-fat diet exposure on learning \& memory. Physiol. Behav. 2015, 152, 363-371.

43. Lv, J.; Qi, L.; Yu, C.; Yang, L.; Guo, Y.; Chen, Y.; Bian, Z.; Sun, D.; Du, J.; Ge, P.; et al. Consumption of spicy foods and total and cause specific mortality: Population based cohort study. BMJ 2015, 351, h3942. [CrossRef] 